\section{ORIGINAL RESEARCH}

J. Narvid

H.M. Do

N.H. Blevins

N.J. Fischbein

\title{
CT Angiography as a Screening Tool for Dural Arteriovenous Fistula in Patients with Pulsatile Tinnitus: Feasibility and Test Characteristics
}

\begin{abstract}
BACKGROUND AND PURPOSE: The diagnosis of intracranial DAVF with noninvasive cross-sectional imaging such as CTA is challenging. We sought to determine the sensitivity and specificity of CTA compared with cerebral angiography for DAVF in patients presenting with PT.
\end{abstract}

MATERIALS AND METHODS: Following approval of the institutional review board, we reviewed all patients who underwent CTA for PT from 2004 to 2009 and collected clinical and imaging data. Seven patients with PT and proved DAVF and 7 age- and sex-matched control patients with PT but no DAVF composed the study group. CTA images were blindly interpreted by 2 experienced neuroradiologists for the presence of 5 variables: asymmetric arterial feeding vessels, "shaggy" appearance of a dural venous sinus, transcalvarial venous channels, asymmetric venous collaterals, and abnormal size and number of cortical veins. Asymmetric attenuation of jugular veins was additionally assessed.

RESULTS: The presence of arterial feeders showed good test characteristics for screening, with a sensitivity of $86 \%(95 \% \mathrm{Cl}, 42-99)$ and a specificity of $100 \%(95 \% \mathrm{Cl}, 52-100)$. A shaggy sinus or tentorium was highly specific: sensitivity of $42 \%(95 \% \mathrm{Cl}, 11-79)$ and specificity of $100 \%(95 \% \mathrm{Cl}$, $56-100)$. The presence of transcalvarial venous channels demonstrated a poor sensitivity of $29 \%$ (95\% $\mathrm{Cl}, 5-70)$ but a high specificity $86 \%(95 \% \mathrm{Cl}, 42-99)$. CT attenuation of the jugular veins showed statistically significant asymmetry in the DAVF group versus the control group $(P<.05)$.

CONCLUSIONS: CTA can be used to screen for DAVF in patients with PT. The presence of asymmetrically visible and enlarged arterial feeding vessels has a high sensitivity and specificity for the diagnosis of DAVF.

\begin{abstract}
ABBREVIATIONS: APA = ascending pharyngeal artery; $\mathrm{Cl}=$ confidence interval; $\mathrm{CTA}=\mathrm{CT}$ angiography; $\mathrm{CTV}=\mathrm{CT}$ venography; $\mathrm{DAVF}=$ dural arteriovenous fistula; $\mathrm{DSA}=$ digital subtraction angiography; $\mathrm{dx}=$ diagnosis; IJV = internal jugular vein; $\mathrm{L}=$ left; $\mathrm{MHT}=$ meningohypophyseal trunk; MIP = maximum intensity projection; $\mathrm{MM}=$ middle meningeal artery; $\mathrm{MRA}=\mathrm{MR}$ angiography; $M R V=M R$ venography; NPV = negative predictive value; Occ = occipital artery; $P A u r=$ posterior auricular artery; PCA = posterior communicating artery; PPV = positive predictive value; $\mathrm{PT}=$ pulsatile tinnitus; $\mathrm{R}=$ right; Vert = vertebral artery
\end{abstract}

$\mathbf{P}$ T is a common clinical symptom. ${ }^{1-5}$ Its causes are predominantly vascular, either arterial or, more commonly, venous. ${ }^{5-9}$ An extensive literature on imaging in PT describes a wide variation in the incidence of structural findings in patients with PT. ${ }^{10,11}$ These discrepancies likely reflect heterogeneity in study populations, radiologist expertise, and imaging technique used in the evaluation. The utility of any first imaging test of PT is dependent on the sensitivity with which it detects treatable or worrisome causes of PT and allows appropriate management to be undertaken. Accordingly, the ideal test would demonstrate high sensitivity for such clinically significant causes of PT. ${ }^{4}$

A recent review of case series found structural/anatomic causes of PT in $44 \%-91 \%$ of cases. ${ }^{9}$ Although a majority of patients may remain "idiopathic" even after extensive workup, the possibility of a morbid and/or treatable cause of PT,

Received April 13, 2010; accepted after revision August 4.

From the Department of Radiology, Division of Neuroradiology (J.N., H.M.D., N.J.F.) and Department of Otolaryngology-Head and Neck Surgery (N.H.B.), Stanford University Medical Center, Stanford, California.

Please address correspondence to Jared Narvid, MD, Department of Radiology, Division of Neuroradiology, 300 Pasteur Dr, Room S047, Stanford, CA 94305-5105; e-mail: jnarvid@stanford.edu

DOI 10.3174/ajnr.A2328 such as a DAVF, represents a common indication for imaging. Hence, there is interest in and the necessity for imaging protocols that might accurately diagnose or exclude such pathology. Various imaging strategies have been proposed, including MR imaging, MRA, MRV, carotid sonography, CTA/CTV, and DSA. ${ }^{12-17}$ None of these studies have well-defined test characteristics. Conventional angiography remains the most sensitive and specific technique for diagnosing DAVF. Thus, patients routinely undergo DSA if DAVF remains in the differential diagnosis for a patient with PT following noninvasive imaging.

Findings of DAVF on DSA are well-described, and the technique is accurate in experienced hands. ${ }^{18}$ The risk of neuroangiography is not negligible, however, and access to experienced neuroangiographers remains limited. ${ }^{24} \mathrm{CT}$ and CTA, on the other hand, are efficient, well-tolerated, widely available, and low risk if performed by using appropriate parameters.

Recently, multidetector CTA has successfully supplanted diagnostic DSA in the evaluation of a variety of vascular lesions. What is not surprising, several authors have suggested its use as a means of detecting DAVF and have proposed imaging findings that might suggest the diagnosis. ${ }^{15,25} \mathrm{CT}$ also carries the advantage of identifying vascular neoplasms as well 
as bony/developmental abnormalities of the middle ear and otic capsule that may lead to PT. Furthermore, it allows assessment of the dural venous sinuses for stenoses or diverticula unrelated to the presence of a dural fistula. To our knowledge, however, only 2 studies have evaluated CTA/CTV in patients with PT. Using CTA/CTV, Krishnan et al ${ }^{16}$ evaluated 16 patients with PT and, somewhat surprising, did not identify a single DAVF. More recently, in a retrospective review of patients with DAVF, Cohen et $\mathrm{al}^{21}$ found a low sensitivity (15.4\%) of CTA for DAVF, but these authors did not describe how the CTAs were evaluated. In this study, we propose and depict a set of CTA findings that suggests the presence of DAVF in patients with PT, and we evaluate the diagnostic performance of these findings as a screening tool for DAVF in patients with PT.

\section{Materials and Methods}

\section{Patient Population}

The study was approved by our institutional review board. The data base of our PACS was searched from January 2003 to September 2009 for patients diagnosed with DAVF. Only patients with both CT and CTA as part of their initial work-up and subsequent confirmatory DSA were included in this analysis. A similar search of our hospital information system was performed to identify patients presenting with PT who also had CTA performed but who were not diagnosed with dural fistula. Electronic medical records were used to gather demographic and clinical information. These search results yielded sets of patients with PT who either did (patients) or did not have (controls) DAVFs. All patients underwent confirmatory conventional angiography. Controls with clinically worrisome PT underwent diagnostic conventional angiography, and those with clinically benign PT underwent CTA and confirmatory MR imaging/MRA. Patients with DAVF without $\mathrm{PT}$ as a first symptom were excluded. Patients with PT without DAVF (cryptogenic or other venous abnormality) were used as controls. Additionally, all patients classified as having PT demonstrated pulse-synchronous tinnitus defined by otologic clinical evaluation.

Seven patients with PT and angiographically proved DAVF and 7 age- and sex-matched controls with PT but no DAVF composed the study group.

\section{CT/CTA/CTV Parameters}

We performed multidetector CT/CTA/CTV on a 16-detector scanner (LightSpeed; GE Healthcare, Milwaukee, Wisconsin). Noncontrast images were obtained from the vertex to the skull base (5-mm section thickness, $120 \mathrm{kV}, 400 \mathrm{~mA})$. This was followed by CTA $(0.625-\mathrm{mm}$ section thickness, $140 \mathrm{kV}, 250 \mathrm{~mA}$ ). Triggering of CTA acquisition was done manually by a technologist based on peak contrast enhancement in the aortic arch following administration of $120 \mathrm{~mL}$ of iohexol with a concentration of $350 \mathrm{mg} \mathrm{I} / \mathrm{mL}$ (Omnipaque 350; GE Healthcare, Princeton, New Jersey) at a rate of $4-5 \mathrm{~mL} / \mathrm{s}$. Images acquired in this manner demonstrated arterial phase opacification but with both arterial and venous vasculature well-opacified, so both arterial and venous structures could be assessed. The CT source images were postprocessed to create contiguous 1-mm coronal and sagittal reformations, as well as axial, coronal, and sagittal MIP images with both 3and $5-\mathrm{mm}$ section thickness. Volume-rendered 3D images and curved planar reformatted images of the bilateral common and inter- nal carotid arteries and vertebral arteries were also created, as well as $3 \mathrm{D}$ images of the vessels of the circle of Willis.

\section{Image Evaluation}

Imaging studies were reviewed independently on a PACS workstation by 2 experienced neuroradiologists who were blinded to the final diagnosis. Images of patients and controls were presented to the blinded reviewers in random order. Only the lead author was aware of the final diagnosis based on DSA. Imaging studies contained source data as well as multiplanar and processed series, and each patient's entire examination was evaluated in the same sequence. Before assessment of findings associated with DAVF, the images were evaluated for the presence of mass lesions, an aberrant internal carotid artery or persistent stapedial artery, and abnormalities of the temporal bone such as dehiscence of the superior semicircular canal.

Imaging studies were evaluated for the presence or absence of 5 variables that are associated with DAVF: numerous, asymmetric, and/or dilated feeding arteries; numerous, asymmetric, and/or dilated venous collaterals; "shaggy" appearance of a dural venous sinus or the tentorium cerebelli; transcalvarial venous channels; and increase in the size and number of cortical veins. These variables were scored as "present" or "absent." Images were additionally analyzed for measurable asymmetry in jugular venous attenuation. Finally, images were also comprehensively evaluated for the presence of any pathology that could explain PT at the time of clinical interpretation as well as subsequently during dedicated research evaluation.

Cervical vessels were evaluated specifically to identify usually asymmetric prominent and/or numerous branches of the external carotid arteries that might serve as DAVF feeders. The arterial nature of these channels was confirmed by assessment of their course, inspection of their origin from identifiable arteries (eg, external carotid branches), and also via depiction of equivalent attenuation to arterial sources. When these criteria were met, the vessels were declared positive for arterial feeders. Conversely, numerous and prominent cervical vessels that appeared venous in course, opacification, and origin were labeled as such.

A shaggy appearance of the sinus or tentorium was determined by inspection of these structures for the presence of irregularity along their margins because venous sinuses are typically smoothly marginated. Irregularity was subjectively assessed and thought to suggest the presence of innumerable small venous efferents and/or intimal thickening associated with venous sinus hypertension.

Because fistulizing arterial feeders from the occipital artery often take a transosseous path at the mastoid foramen alongside emissary veins located within or adjacent to the occipitomastoid suture, we evaluated the bone around the occipitomastoid suture for significant asymmetry in the size and number of regional vascular grooves or channels.

Asymmetric attenuation of jugular veins was assessed in a fashion similar to that of previously reported methodology. ${ }^{25}$ Hounsfield measurements were made on both patients and controls in 4-mm regions of interest centered within both jugular veins at a level $1 \mathrm{~cm}$ below the jugular foramen. Three measurements of mean attenuation were made, and the average value was used for analysis. All measurements were made below the level of stenosis in patients with DAVFrelated dural venous sinus or jugular stenosis.

After evaluation of CT/CTA datasets, the participating neuroradiologists were unblinded. Neuroangiographic datasets were evaluated on the same PACS workstation used for the prior analysis. The presence of abnormal connections between arterial feeders and a dural 


\begin{tabular}{|c|c|c|c|c|c|c|}
\hline Patient & $\begin{array}{c}\text { Age }(y r) / \\
\text { Sex }\end{array}$ & Arterial Supply & $\begin{array}{c}\text { Venous } \\
\text { Drainage }\end{array}$ & Stenoses & $\begin{array}{l}\text { Cortical } \\
\text { Venous }\end{array}$ & $\begin{array}{c}\text { Cognard } \\
\text { Stage } \\
\end{array}$ \\
\hline 1 & $46 / F$ & R Occ/R Vert/R APA & Marginal & None & No & Ila \\
\hline 2 & $46 / F$ & R PCA/R Vert/R Occ/R MM/R APA/L Occ & Sigmoid & R Jugular & No & Ila \\
\hline 3 & $79 / F$ & $\mathrm{R}$ MM/R Occ/R MHT & Occipital & R Jugular & No & I \\
\hline 4 & $55 / M$ & R Occ/R MHT/R MM & Sigmoid & R Jugular & No & Ila \\
\hline 5 & $43 / \mathrm{M}$ & R Occ/R PAur/R MHT & Marginal & None & No & I \\
\hline 6 & $52 / F$ & L Occ/L Vert & Marginal & None & No & Ila \\
\hline 7 & $40 / F$ & $\mathrm{R} \mathrm{MM} / \mathrm{R}$ APA/R Occ & Sigmoid & R Sigmoid & Yes & $\| \mathrm{la}+\mathrm{b}$ \\
\hline
\end{tabular}

venous sinus or leptomeningeal vein constituted diagnostic criteria for DAVF. All DAVFs were classified according to the Cognard stage on the basis of conventional angiography: type I representing fistula confined to the sinus wall with antegrade flow; type IIA, a fistula with retrograde flow within the sinus; type IIB, a fistula with retrograde reflux into cortical veins; type IIA $+B$, a fistula with retrograde flow within the sinus and reflux into cortical veins; type III, a fistula with direct drainage into cortical veins; type IV, a fistula with reflux into ectatic cortical veins; and type $\mathrm{V}$, a fistula with spinal perimedullary drainage.

\section{Statistical Analysis}

The 5 imaging features described above were used as discriminant criteria. We thus based the performance of CT/CTA on its ability to discriminate patients from controls as measured by sensitivity and specificity. ${ }^{26}$ Five of the discriminant criteria relied on the judgment of neuroradiologists, while the sixth imaging feature was based on objective measurements of CT attenuation of the draining jugular veins. These latter measurements were subject to standard paired 2-tailed Student $t$ test to assess asymmetry in jugular opacification in both groups. A $P$ value $\leq .05$ was considered a significant difference. We calculated $95 \%$ CIs according to the efficient score method.

\section{Results}

Seven patients underwent CT/CTA for diagnostic work-up of PT and were found to have DAVF on subsequent DSA (Table 1). The average age was 48.9 years (range, $40-79$ years). There were 5 women and 2 men. Six of the 7 had PT on the right and were found to have predominantly a right-sided supply and right-sided drainage. One patient had left-sided supply and drainage. One patient had a bilateral supply that drained into the right sigmoid sinus. Drainage patterns were variable, with 3 patients demonstrating a fistula at the sigmoid sinus, 3 patients demonstrating fistula at the marginal sinus, and 1 patient demonstrating a fistula to an occipital sinus. In comparison and in keeping with the general population, 6 of 7 patients demonstrated right-sided dominant drainage with 1 demonstrating codominant transverse sinus/jugular drainage. All fistulas were evaluated for Cognard stage and venous drainage. Four of 7 patients demonstrated venous sinus stenosis. All stenoses were downstream of the fistula in either the high jugular vein (3/4) or sigmoid sinus (1/4). These stenoses did not correlate with other signs of venous hypertension such as cortical venous drainage. Four of 7 patients demonstrated Cognard stage IIa with some retrograde flow through the venous sinuses. Two patients showed Cognard stage I fistula with antegrade venous flow. One patient showed stage IIa $+b$ with evidence of retrograde venous sinus flow in addition to cortical venous drainage. This patient reported a long-standing his-

\begin{tabular}{lccc}
\hline \multicolumn{4}{l}{ Table 2: Clinical characteristics of controls (PT without DAVF) } \\
\hline Patient & $\begin{array}{c}\text { Age }(y r) / \\
\text { Sex }\end{array}$ & Symptoms & CTA Findings \\
\hline A & $53 / F$ & R PT & Sigmoid sinus diverticulum \\
B & $30 / F$ & R PT & No dx \\
C & $53 / F$ & R PT & Transverse sinus stenosis \\
D & $48 / F$ & L PT & No dx \\
E & $39 / F$ & R PT & No dx \\
F & $33 / M$ & L PT & No dx \\
G & $75 / M$ & R PT & No dx \\
\hline
\end{tabular}

tory of right-sided PT before hospitalization for intracerebral hemorrhage.

Age- and sex-matched controls averaged 41.3 years of age (range, 30-75 years). All presented with PT (Table 2), with predominantly right-sided (5/7) symptoms as in our patients (6/7). Most controls (5/7) had no evidence of pathology demonstrated on CTA images. Two patients had findings on CTA suggesting a possible etiology of symptoms. One patient had a venous diverticulum of the sigmoid sinus ipsilateral to the PT, and the other patient had moderate stenosis of the ipsilateral transverse sinus.

Of 7 patients with DAVFs, 6/7 demonstrated asymmetrically numerous and enlarged arterial feeders surrounding the ipsilateral carotid artery and its external branches (Table 3 and Fig 1). None of the controls demonstrated this finding. The presence of asymmetrically increased arterial feeders (Table 3) showed good test characteristics for screening, with a sensitivity of $86 \%$ (95\% CI, 42-99), a specificity of $100 \%$ (95\% CI, $52-100)$, PPV of $100 \%$ (95\% CI, 56-100), and NPV of $88 \%$ (95\% CI, 67-88). The appearance of asymmetric feeders at the neck and skull base on CTA matched the pattern of arterial supply from external carotid artery branches on angiography. For example, an enlarged occipital artery seen on CTA would also be seen on conventional angiography. Nevertheless, 3/7 patients had angiographic evidence of supply from the MHT (ie, a small vessel that was not consistently identifiable on CTA).

In contradistinction, $3 / 7$ cases demonstrated numerous venous collaterals in the neck (Fig $1 B$ ). Likewise, 2/7 controls showed this finding, thereby generating a sensitivity of $42 \%$ (95\% CI, 11-79), specificity of 71\% (95\% CI, 30-95), PPV of $60 \%$ (95\% CI, 17-93), and NPV of 56\% (95\% CI, 38-70). Its diagnostic performance thus did not prove useful in discriminating patients from controls. The presence of transcalvarial channels also proved a relatively poor sign, with $2 / 7$ patients and $1 / 7$ controls demonstrating this finding (Fig 1C). The presence of transcalvarial venous channels demonstrated a poor sensitivity of $29 \%$ (95\% CI, 5-70) but a high specificity of 


\begin{tabular}{|c|c|c|c|c|c|c|c|c|}
\hline CTA Finding & $\begin{array}{c}\text { Sensitivity } \\
(\%)\end{array}$ & $95 \% \mathrm{Cl}$ & $\begin{array}{c}\text { Specificity } \\
(\%)\end{array}$ & $95 \% \mathrm{Cl}$ & $\begin{array}{l}\text { PPV } \\
(\%)\end{array}$ & $95 \% \mathrm{Cl}$ & $\begin{array}{l}\text { NPV } \\
(\%)\end{array}$ & $95 \% \mathrm{Cl}$ \\
\hline Asymmetrically increased arterial feeders & 86 & $42-99$ & 100 & $52-100$ & 100 & $56-100$ & 88 & $67-88$ \\
\hline Asymmetrically increased venous collaterals & 42 & $11-79$ & 71 & 30-95 & 60 & $17-93$ & 56 & $38-70$ \\
\hline Transcalvarial channels & 29 & $5-70$ & 86 & $42-99$ & 67 & $12-98$ & 55 & $43-62$ \\
\hline Shaggy sinus or tentorium & 42 & $11-79$ & 100 & 56-100 & 100 & $31-100$ & 64 & $55-64$ \\
\hline Prominent cortical venous drainage & 29 & $5-70$ & 71 & 30-95 & 50 & 9-90 & 50 & $35-63$ \\
\hline
\end{tabular}
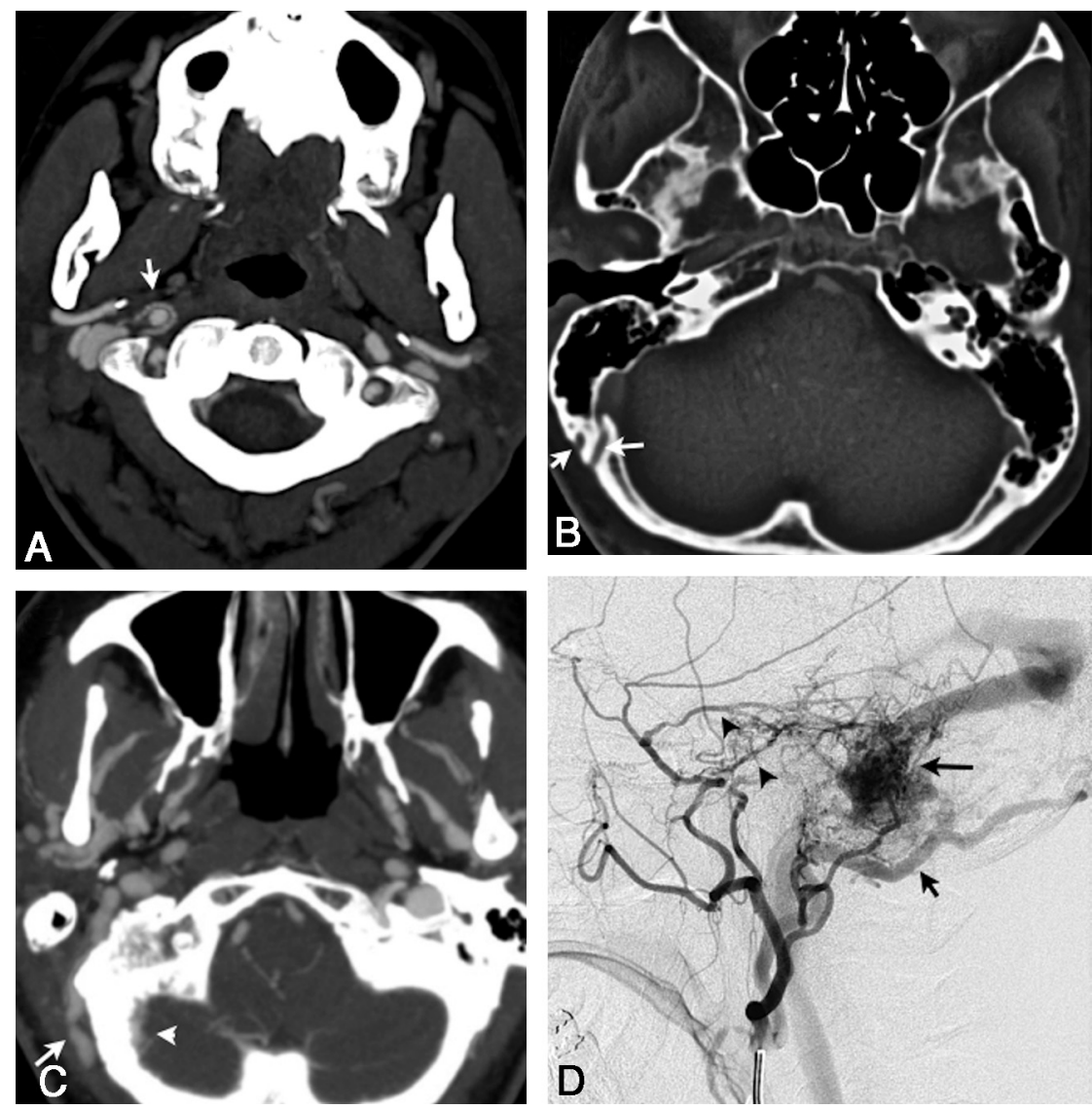

Fig 1. A, Axial 3-mm-slab MIP from CTA. A clearly abnormal "ring" of arterial feeders is seen around the right distal cervical carotid artery at the C1-2 level (white arrow) in a patient with a right sigmoid DAVF (patient 1). B, Large transosseous venous channels (white arrows) are present on the right in patient 1 . This is an uncommon but specific finding for DAVF. These channels do not track to the occipitomastoid suture, a structure from which they must be distinguished in some cases by viewing serial sections. $C$, Markedly asymmetric venous collaterals along the right occipital bone, both intracranially (white arrow) and extracranially (white arrowhead), as well as asymmetrically enlarged and numerous veins throughout the infratemporal fossa (patient 2). This patient also had large transosseous collaterals (not shown). D, DSA, arterial phase, right external carotid injection in a lateral projection in patient 2, shows arterial feeders from the markedly enlarged occipital artery (short black arrow), as well as from the middle meningeal artery (black arrowheads), the correlates of the arterial feeders shown on the CTA in $C$, with a sigmoid DAVF (long black arrow).

86\% (95\% CI, 42-99), PPV of 67\% (95\% CI, 12-98), and NPV of 55\% (95\% CI, 43-62) (Table 3).

A majority of patients with DAVFs (4/7) demonstrated a shaggy appearance of the tentorium or a venous sinus (Fig $2 C-E)$. This finding, also seen on angiography, represents a conglomeration of pathologic sequelae implicated in the angiogenic process. The opening of dural vascular channels, intimal thickening and stenosis, venous hypertension, and dilation of and reflux into cortical veins may all contribute to this appearance. Because no controls showed this sign, it had excellent diagnostic performance (Table 3 ). A shaggy sinus or tentorium was highly specific: sensitivity of $42 \%$ (95\% CI, 1179), a specificity of $100 \%$ (95\% CI, 56-100), PPV of $100 \%$ (95\% CI, 31-100), and NPV 64\% (95\% CI, 55-64).
Although the presence of cortical venous drainage is a critical prognostic finding on angiography, CTA does not permit subtle distinction regarding flow directionality. We hypothesized that anatomic depiction of cortical venous dilation may represent a CTA correlate. Indeed, 2 patients demonstrated striking venous collateralization on CTA. Nevertheless, only $2 / 7$ cases demonstrated this finding, generating a sensitivity of $29 \%$ (95\% CI, 11-29), specificity of $100 \%$ (95\% CI, 82-100), PPV of $100 \%$ (95\% CI, 38-90), and NPV of $58 \%$ (95\% CI, 49-58). CT attenuation of the jugular veins, measured in Hounsfield units $1 \mathrm{~cm}$ below the skull base (Fig 3), showed a statistically significant asymmetry in the DAVF group versus the control group $(P<.05)$. 

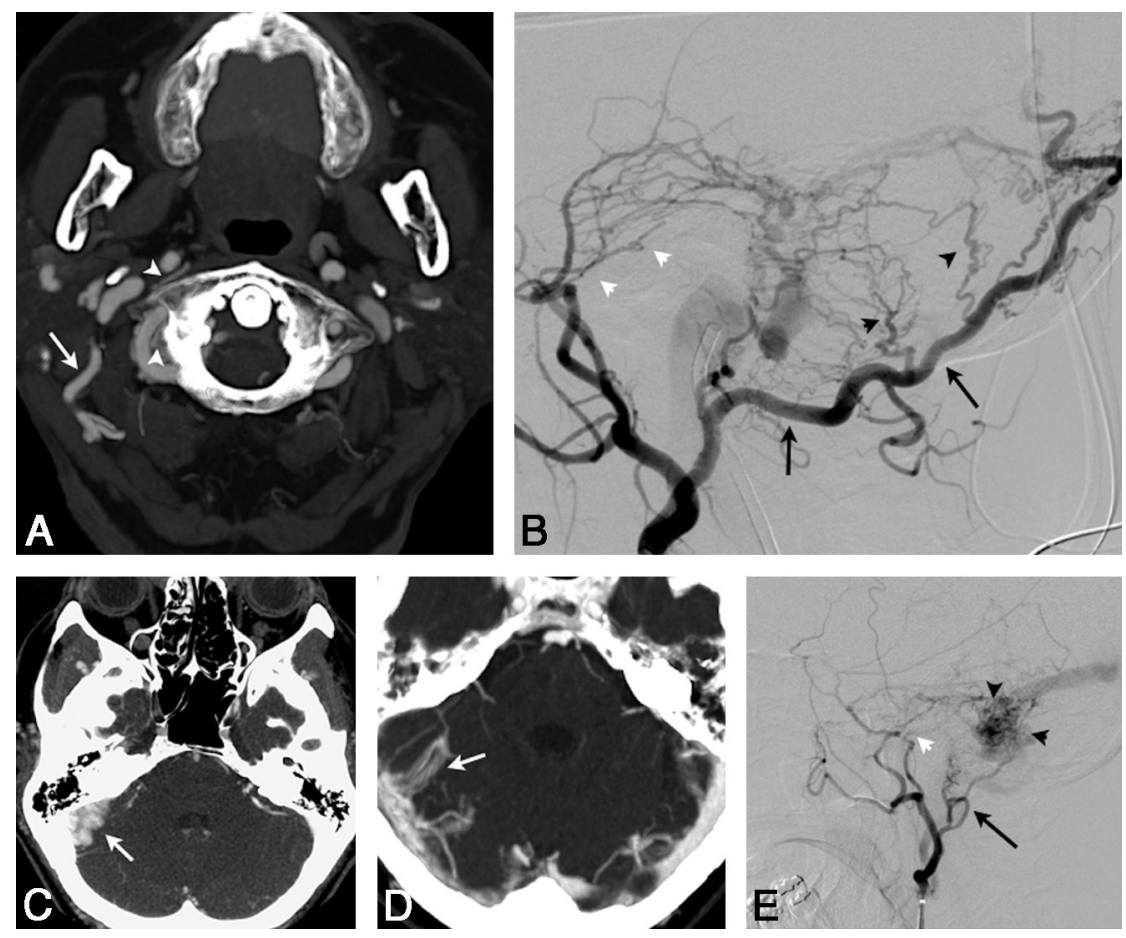

Fig 2. A, Axial 3-mm-slab MIP from CTA in patient 3 with a right sigmoid DAVF exhibits a markedly enlarged $\mathrm{R}$ occipital artery at the C1-2 level (large white arrow). There are also abnormally prominent small vessels surrounding the right vertebral and distal internal carotid arteries (white arrowheads). $B$, DSA, arterial phase, right external carotid injection in a lateral projection, demonstrates a markedly enlarged occipital artery (long black arrows), the angiographic correlate of the CTA in A. Transosseous branches (black arrowheads) of the occipital artery and middle meningeal artery feeders (white arrowheads) contribute to this sigmoid dural fistula. C, Axial 3-mm-slab MIP. The right sigmoid sinus is markedly enlarged and has a very irregular medial margin (arrow), an example of the shaggy sinus sign (patient 2). D, Another shaggy sinus sign involving the right transverse sinus. (The shaggy sinus only occurred in patients and was not seen in the control population.) E, DSA, right external carotid injection in a lateral projection, shows a typical DAVF with abnormal early opacification of the proximal occluded sigmoid sinus with retrograde flow through the transverse sinus to the contralateral dural sinuses. Arterial feeders arise from the middle meningeal artery (white arrowhead) and posterior auricular artery (black arrow) in patient 2. Angiographically, this sinus also appears shaggy (black arrowheads).
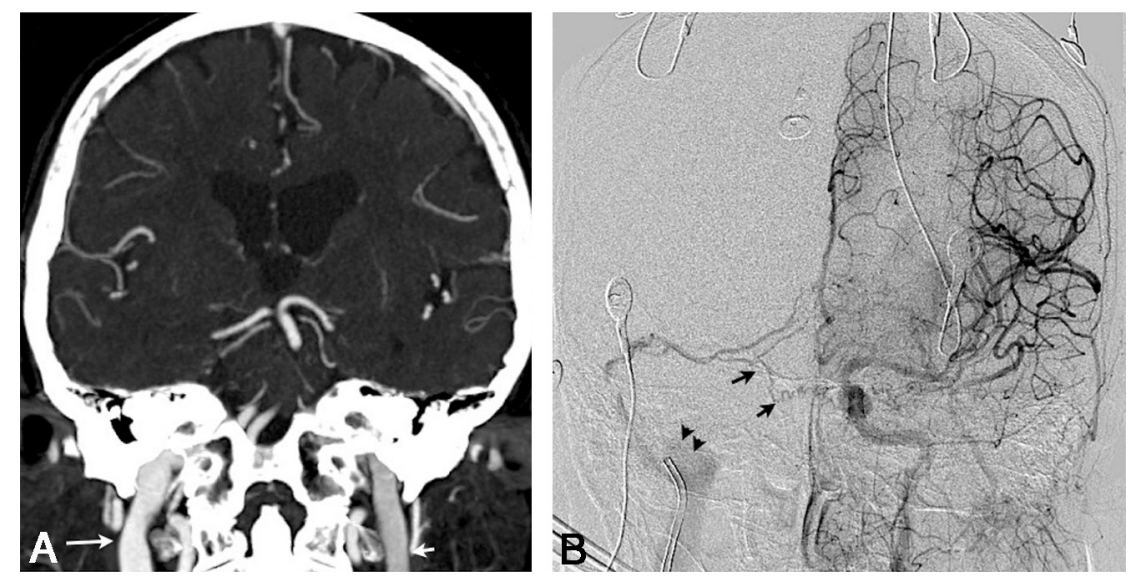

Fig 3. A, Coronal 3-mm-slab MIP from CTA in patient 3 with a right transverse sinus DAVF, predominantly supplied by right occipital, middle meningeal, and marginal tentorial branches; some supply from the left internal carotid is also seen, as described below. This case illustrates the "asymmetric jugular attenuation" sign, which is easily appreciated when the $\mathrm{R}$ and L IJVs are compared. The attenuation of the R IJV is $402 \mathrm{HU}$, and the L IJV is $318 \mathrm{HU}$. B, DSA, late arterial phase, left internal carotid injection in an anteroposterior projection in same patient as in A. Fistula supply from small left internal carotid artery dural and tentorial branches (black arrows) contributes to early opacification of the right sigmoid sinus and IJV (note the catheter near the jugular bulb). Due to the arteriovenous shunt surgery and drainage pattern, iodinated contrast preferentially fills the right jugular vein (black arrowheads) and leads to an asymmetric right-sided increase in CT attenuation, which is seen in $A$.

\section{Discussion}

The initial evaluation of the patient with PT begins with a careful history and otoscopic evaluation. The presence of a mass on otoscopy warrants temporal bone CT for further evaluation. PT in the face of normal otoscopic findings, however, represents a common otologic diagnostic dilemma. The differential diagnosis remains broad and unfortunately includes potentially serious vascular etiologies such as DAVF and more benign vascular etiologies such as venous stenoses and diverticula. Moreover, the literature has many statements such as "if DAVF is likely, then catheter-angiography is warranted,"16 which presume some clinical symptom or sign unique to this diagnosis. The otology literature has described this dilemma, ${ }^{18}$ with some advocating liberal use of angiography, while others advance MR imaging and CT-based approaches. ${ }^{4,6}$ Clinical evaluation clarifies the presence of subjective "whooshing" 

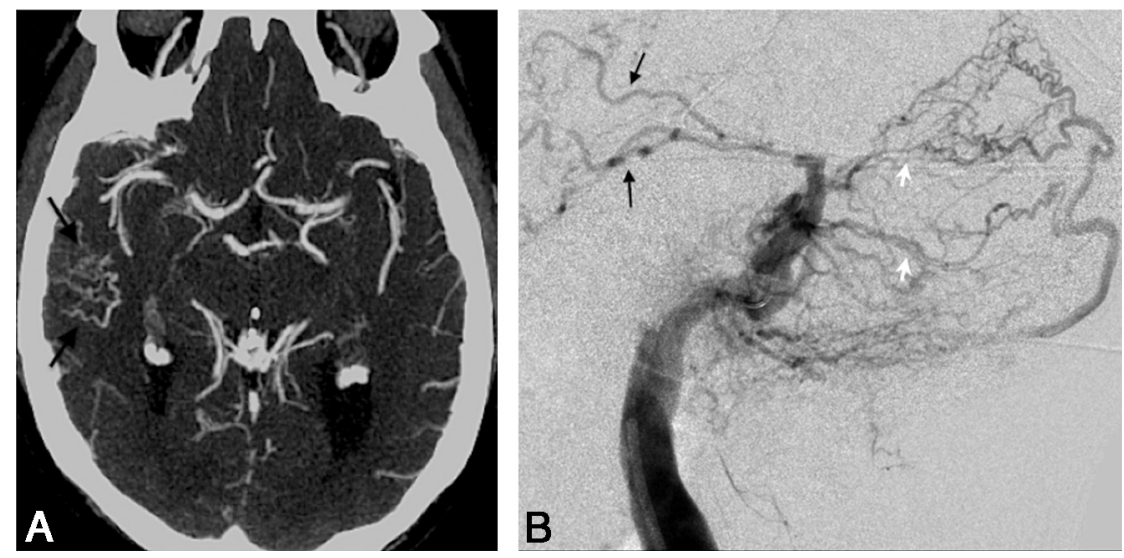

Fig 4. A, Axial 3-mm-slab MIP from CTA in patient 7 illustrates a cluster of abnormally prominent, tortuous, and numerous veins overlying the right temporal lobe (black arrows). These are dilated cortical venous efferents, which are thought to confer increased risk of hemorrhage. $B$, DSA, right external carotid injection, venous phase, in a lateral projection in the same patient as in $A$, demonstrates retrograde cortical or leptomeningeal draining veins (black arrows) in the right temporal region in this Cognard lla $+b$ fistula. Note multiple arterial feeders arising from the occipital artery (white arrows).

pulsations that extinguish with gentle neck pressure or altered head position, a finding that supports more benign venous origins of PT. Alternatively, the presence of a bruit raises concern for an arterial vascular abnormality, which indicates complete imaging including DSA. Moreover, the impact of PT on the well-being of the patient is a significant factor in determining how aggressive the work-up should be. The present data provide guidance for most patients who present with neither obvious benignity/low impact nor a clear and clearly worrisome audible bruit.

CTA analysis in the current study was based on cross-sectional correlates of classic angiographic findings of DAVF. These findings are based on the sine qua non of DAVF: arteriovenous shunting. Angiographically, anomalous connections between dural arteries and venous sinuses can be seen directly. Ancillary findings relate to the presence of angiogenic activity and the end results of arterial pressure on venous structures. These findings include multiple arterial feeders, venous intimal thickening, stenosis and/or thrombosis, and dilation of and reflux within venous efferents. ${ }^{19,20,27}$

The hypothesis that additional potential CTA findings may demonstrate improved test characteristics derives from the above-mentioned angiographic experience. Two indirect characteristics have been previously reported to be associated with DAVF on CT or CTA: transcalvarial venous channels and asymmetric attenuation of jugular veins. We also chose to assess numerous asymmetric and/or dilated arteries; numerous asymmetric and/or dilated venous collaterals; shaggy appearance of a dural venous sinus or the tentorium cerebelli; and increased number and/or enlargement of cortical veins, all of which derive from angiographic findings.

MR imaging-based approaches have demonstrated some success in evaluating DAVF. ${ }^{21,28,29}$ Studies have used a variety of techniques and sequences and have shown variable diagnostic performance. De Marco et $\mathrm{al}^{28}$ presented some of the earliest data, finding MR imaging cortical vein dilation and venous occlusion in 8 of 12 patients with angiographically proved DAVF by using basic spin- and gradient-echo sequences. This description is contrary to the present experience in which the presence of cortical venous dilation was relatively rare, $2 / 7$. Such discrepancies can be accounted for by differ- ences in the study population, a small cohort of DAVF not limited to those presenting with $\mathrm{PT}$, and differences in technique.

More recent MR angiographic analyses have yielded a remarkably wide range of results with sensitivities from $50 \%$ to $100 \% .^{21,29}$ Time-resolved MR imaging and 4D flow MR imaging have shown promise in providing angiography-like images $^{30,31}$ and in providing the time resolution that routine CTA/CTV and MR imaging/MRA/MRV lack. Yet these studies represent the results of highly specialized and often proprietary MR images, which, as such, remain in limited use. As shown in the current study, CTA can provide equivalent diagnostic performance but with improved osseous. Its additional merits in cost, efficiency, and widespread availability hardly need emphasis. Recent data on the comparative strength of CTA in evaluating alternative etiologies to PT, such as glomus tumors, buttress the argument that CTA can provide an efficient and comprehensive first-step assessment of patients with $\mathrm{PT}^{38}$

The additional radiation associated with CT angiography in comparison with noncontrast CT of the head has been recently investigated. ${ }^{39}$ Standard parameters yield mean effective doses of $2.7 \mathrm{mSv}$ for nonenhanced CT, while CTA delivers $5.4 \mathrm{mSv}$. As a comparison, however, both studies fall below the relatively commonplace 8 - to 9-mSv dosage of an abdominal CT. Moreover, cerebral angiography commonly averages 10.6 $\mathrm{mSv}$ in effective dose equivalent. ${ }^{40}$ The detailed anatomic data provided by CTA and the possible avoidance of DSA dosage must be balanced against the increased dose delivered by CTA.

CTA demonstrates a number of direct and indirect signs that are suggestive of intracranial DAVF. These signs can be used to screen for DAVF in patients with PT. In particular, the presence of abnormally enlarged arterial feeding vessels, a finding not emphasized in prior literature, has high sensitivity, specificity, and PPV for the diagnosis of DAVF.

The discrepancy between the current results and the findings of Cohen et al, ${ }^{21}$ however, does require further discussion. In that series of 46 patients, 13 patients underwent CTA, only 2 of whom had findings positive for DAVF. Yet on what basis DAVF was excluded remains speculative because this study was a chart review in which images were not assessed by using 
a standard set of criteria. It may be that this low sensitivity (15\%) represents only a "standard" evaluation without specific focus on potential diagnostic features of DAVF. Because this is often what referring physicians receive, they are then left to wonder whether CTA is worth ordering at all.

With regard to the limitations of our study, our findings may not be easily replicated in all practice settings. The process of formalized evaluation in the academic setting may represent an idealized evaluation. Regardless, the objectives of this study are not only the evaluation of diagnostic performance of CTA in DAVF diagnosis but also the hypothesis-driven depiction of radiographic signs. Here, the appearance of abnormally numerous and enlarged arterial feeders, shaggy sinuses and tentorium, and asymmetric sinus attenuation helps provide keys to improved radiologic evaluation. These signs, especially if confirmed by other investigators, will allow radiologists, neuroradiologists, and experienced nonradiologist physicians to increase their diagnostic sensitivity and specificity for DAVF. Our limited CTA depiction of cortical venous collaterals is likely an artifact of selection bias. In choosing to evaluate patients with PT rather than intracranial hemorrhage, we accordingly selected a cohort with lower grade DAVFs that, by nature, less frequently demonstrate cortical venous collaterals.

A potential additional limitation involves the use of PPV because it depends on the prevalence of disease within the study group. In a case-control study such as this one, the population is necessarily enriched with disease. Nevertheless, as noted by Madani and Connor, ${ }^{9}$ the prevalence of DAVF in patients with PT is variably reported to be as high as $20 \%$. A study population with a prevalence of $50 \%$ DAVF necessarily will accentuate the PPV.

Interest in cross-sectional correlates of angiographic findings has grown with the advent of increased CT angiographic gantry speed and pitch, improved section profile, ever-advancing tools for postprocessing and image manipulation, and conebeam technologies. In fact, experience in evaluating DAVF with C-arm CT, as recently described by Hiu et $\mathrm{al}^{17}$ and Lasjaunias et al, ${ }^{33}$ helps generate confidence in evaluating CTA correlates of classic angiographic findings. ${ }^{32}$

The current series supports findings of earlier reports that suggest that DAVFs associated with PT often involve the sigmoid sinus. ${ }^{5,33,34} \mathrm{McDougall}^{\text {et }} \mathrm{al}^{35}$ provided a specific description of a cohort of patients with DAVF at the marginal sinus, in which 11 of 14 presented with PT. The marginal sinus is located between layers of dura that encircle the foramen magnum, communicating with the basal venous plexus of the clivus anteriorly and the occipital sinus posteriorly. Our series confirms the association of sigmoid sinus and marginal sinus DAVF with PT.

Recent data on the prognosis of nonhemorrhagic DAVF have highlighted the reassuring risk profile of Cognard I and II fistulas. $^{22,36,37}$ Awad et al, ${ }^{34}$ in an early meta-analysis, found 203 patients with PT-associated DAVF showing a nonaggressive natural history. Controversy exists as to the rate at which low-grade fistulas convert to aggressive phenotypes. ${ }^{23,37} \mathrm{CTA}$ evaluation of cortical venous reflux in the current study was limited for several reasons. The cohort of patients with PT does not, in general, have high-grade fistulas. In fact, angiography confirmed no fistula above Cognard grade IIa $+\mathrm{b}$, implying absence of any venous ectasia. Neuroradiologists could not distinguish the presence or absence of cortical venous reflux by using only static anatomic CTA data, given the known lack of marked cortical venous dilation. Thus, a PPV as low as $50 \%$ reflects this limitation. In addition, our series identifies a single case of a patient who personally recalled months of ipsilateral PT symptoms before presenting with intracranial hemorrhage. This single case serves as a reminder that the presence of PT is no guarantee of benign prognosis.

\section{Conclusions}

The patient with PT frequently undergoes cross-sectional imaging evaluation, but there is a wide variation in the imaging methods used and in reported performance of these modalities for the diagnosis of DAVF. CTA provides a convenient low-risk method for additional evaluation of vascular causes. The present data suggest that the combined signs of abnormally prominent arterial feeders, shaggy sinus/tentorium, and asymmetric jugular venous attenuation produce useful diagnostic information with a combined sensitivity and specificity $\geq 90 \%$. The ability of conventional angiography to evaluate venous efferents remains an important diagnostic benefit of DSA because it carries prognostic significance. DSA accordingly is still indicated if CTA is negative but clinical suspicion of DAVF is high. Furthermore, angiographic analysis allows assessment of the feasibility of endovascular therapeutic approaches and the mechanism to accomplish this therapy once a DAVF has been diagnosed noninvasively. We suggest that screening CTA, assessed systematically for the above-discussed variables, should be considered as the first-choice imaging technique to assess structural, neoplastic, and venous causes of PT and to screen for DAVF.

\section{References}

1. Hardison JE, Smith RB, Crawley IS, et al. Self-heard venous hums. JAMA 1981;245:1146-47

2. Chandler JR. Diagnosis and cure of venous hum tinnitus. Laryngoscope 1983;93:892-95

3. Sismanis A. Pulsatile tinnitus. Otolaryngol Clin North Am 2003;36:389-402, viii

4. Vattoth S, Shah R, Cure JK. A compartment-based approach for the imaging evaluation of tinnitus. AJNR Am J Neuroradiol 2010;31:211-18

5. Anderson JE, Teitel D, Wu YW. Venous hum causing tinnitus: case report and review of the literature. Clin Pediatr (Phila) 2009;48:87-88. Epub 2008 Jul 14

6. Branstetter BF, Weissman JL. The radiologic evaluation of tinnitus. Eur Radiol 2006; 16:2792-802

7. De Ridder D, Menovsky T, Van de Heyning P. An otoneurosurgical approach to non-pulsatile and pulsatile tinnitus. B-ENT 2007;3(suppl 7):79-86

8. Sonmez G, Basekim CC, Ozturk E, et al. Imaging of pulsatile tinnitus: a review of 74 patients. Clin Imaging 2007;31:102-08

9. Madani G, Connor SE. Imaging in pulsatile tinnitus. Clin Radiol 2009;64:319-28

10. Waldvogel D, Mattle HP, Sturzenegger M, et al. Pulsatile tinnitus: a review of 84 patients. J Neurol 1998;245:137-42

11. Sismanis A. Pulsatile tinnitus: a 15-year experience. Am J Otol 1998;19:472-77

12. Malek AM, Halbach VV, Dowd CF, et al. Diagnosis and treatment of dural arteriovenous fistulas. Neuroimaging Clin N Am 1998;8:445-68

13. Shah SB, Lalwani AK, Dowd CF. Transverse/sigmoid sinus dural arteriovenous fistulas presenting as pulsatile tinnitus. Laryngoscope 1999;109:54-58

14. Biondi A, Casasco A, Houdart E, et al. Evolution of angiographic signs of venous hypertension and clinical signs of intracranial hypertension in intracranial dural arteriovenous fistulas [in French]. J Neuroradiol 1999;26:49-58

15. Alatakis S, Koulouris G, Stuckey S. CT-demonstrated transcalvarial channels diagnostic of dural arteriovenous fistula. AJNR Am J Neuroradiol 2005;26:2393-96

16. Krishnan A, Mattox DE, Fountain AJ, et al. CT arteriography and venography in pulsatile tinnitus: preliminary results. AJNR Am J Neuroradiol 2006;27:1635-38

17. Hiu T, Kitagawa N, Morikawa M, et al. Efficacy of DynaCT digital angiography in the detection of the fistulous point of dural arteriovenous fistulas. AJNR Am J Neuroradiol 2009;30:487-91 
18. Shin EJ, Lalwani AK, Dowd CF. Role of angiography in the evaluation of patients with pulsatile tinnitus. Laryngoscope 2000;110:1916-20

19. Castaigne $\mathrm{P}$, Bories J, Brunet $\mathrm{P}$, et al. Arteriovenous fistulae of the dura mater: clinical and radiological study of 13 cases [in French]. Ann Med Interne (Paris) 1975;126:813-17

20. Cognard C, Gobin YP, Pierot L, et al. Cerebral dural arteriovenous fistulas: clinical and angiographic correlation with a revised classification of venous drainage. Radiology 1995;194:671-80

21. Cohen SD, Goins JL, Butler SG, et al. Dural arteriovenous fistula: diagnosis, treatment, and outcomes. Laryngoscope 2009;119:293-97

22. Söderman M, Pavic L, Edner G, et al. Natural history of dural arteriovenous shunts. Stroke 2008;39:1735-39

23. Zipfel GJ, Shah MN, Refai D, et al. Cranial dural arteriovenous fistulas: modification of angiographic classification scales based on new natural history data. Neurosurg Focus 2009;26:E14

24. Fifi JT, Meyers PM, Lavine SD, et al. Complications of modern diagnostic cerebral angiography in an academic medical center. J Vasc Interv Radiol 2009;20:442-47

25. Meckel S, Lovblad KO, Abdo G, et al. Arterialization of cerebral veins on dynamic MDCT angiography: a possible sign of a dural arteriovenous fistula. AJR Am J Roentgenol 2005;184:1313-16

26. Chang PJ. Bayesian analysis revisited: a radiologist's survival guide. AJR Am J Roentgenol 1989;152:721-27

27. Lawton MT, Jacobowitz R, Spetzler RF. Redefined role of angiogenesis in the pathogenesis of dural arteriovenous malformations. J Neurosurg 1997;87:267-74

28. De Marco JK, Dillon WP, Halbach VV, et al. Dural arteriovenous fistulas: evaluation with MR imaging. Radiology 1990;176:193-99

29. Noguchi K, Melhem ER, Kanazawa T, et al. Intracranial dural arteriovenous fistulas: evaluation with combined 3D time-of-flight MR angiography and MR digital subtraction angiography. AJR Am J Roentgenol 2004;182:183-90

30. Farb RI, Agid R, Willinsky RA, et al. Cranial dural arteriovenous fistula: diagnosis and classification with time-resolved MR angiography at 3T. AJNR Am J Neuroradiol 2009;30:1546-51

31. Nishimura S, Hirai T, Sasao A, et al. Evaluation of dural arteriovenous fistulas with $4 \mathrm{D}$ contrast-enhanced MR angiography at 3T. AJNR Am J Neuroradio 2010;31:80-85

32. Paolini S, Lanzino G, Colonnese C, et al. Three-dimensional computed tomography angiography in presurgical planning for treatment of infratentorial dural arteriovenous fistulas. J Neurosurg 2009;110:85-89

33. Lasjaunias $\mathrm{P}$, Chiu M, Ter Brugge K, et al. Neurological manifestations of intracranial dural arteriovenous malformations. J Neurosurg 1986;64:724-30

34. Awad IA, Little JR, Akarawi WP, et al. Intracranial dural arteriovenous malformations: factors predisposing to an aggressive neurological course. J Neurosurg 1990;72:839-50

35. McDougall CG, Halbach VV, Dowd CF, et al. Dural arteriovenous fistulas of the marginal sinus. AJNR Am J Neuroradiol 1997;18:1565-72

36. Strom RG, Botros JA, Refai D, et al. Cranial dural arteriovenous fistulae: asymptomatic cortical venous drainage portends less aggressive clinical course. Neurosurgery 2009;64:241-47, discussion 247-48

37. Soderman M, Pavic L, Edner G, et al. Natural history of dural arteriovenous shunts. Stroke 2009;40:e412, author reply e413-14

38. Christie A, Teasdale E. A comparative review of multidetector CT angiography and MRI in the diagnosis of jugular foramen lesions. Clin Radio 2010;65:213-17

39. Mnyusiwalla A, Aviv R, Symons. Radiation dose from multidetector row CT for acute stroke. Neuroradiology 2009;51:635-40

40. Feygelman VM, Huda W, Peters KR. Effective dose equivalents to patients undergoing cerebral angiography. AJNR Am J Neuroradiol 1992;13:845-49 